\title{
La fenomenología del lenguaje y el concepto de la razón práctica en el pensamiento de Charles Taylor
}

\author{
Phenomenology of LANGUAGE AND the CONCEPT OF PRACTICAL REASON IN THE THOUght OF CHARLES \\ TAYLOR
}

Dr. Carlos Medina (carlos.medina@uv.cl) Facultad de Ciencias Económicas y Administrativas, Universidad de Valparaíso (Valparaíso, Chile)

\begin{abstract}
Taking as a starting point Taylor's concept about man as a being of meanings, this article examines, in particular, the way that Taylor elaborates his conception of the practical use of reason, recovering some fundamental notions of the phenomenological tradition and hermeneutics, relating to language, such as, the idea of the background, and the incarnated situation of man. Considering that, ultimately, the background is a horizon of previous reference, from the ontological point of view, to the subjective domain of reason in the human agent, the analysis of Taylor ends up favoring certain approaches of contemporary philosophy such as, Heidegger's existential analytic that aims at leaving aside, paradoxically, the premise of the subject. If we consider this indication, a challenge that remains, then, to the future development of the ways of understanding moral philosophy and philosophical anthropology, is how to link the analysis of the moral problem, in a subject of senses, with the ontological theory of the background that is, however, a-subjective.
\end{abstract}

Key words: Taylor, phenomenology, language, practical reason, background.

\section{Resumen}

Tomando como punto de partida el concepto que tiene Taylor acerca del ser humano como un ser de significados, este artículo estudia el modo como Taylor elabora su concepción del uso práctico de la razón, recuperando ciertas nociones fundamentales de la tradición fenomenológica y hermenéutica relativas al lenguaje, como lo son la idea del trasfondo y la situación encarnada del hombre. Puesto que, en definitiva, el trasfondo constituye un horizonte de referencia anterior, desde el punto de vista ontológico, al dominio subjetivo de la razón en el agente humano, el análisis de Taylor termina por favorecer ciertos enfoques de la filosofía contemporánea como, por ejemplo, la analítica existenciaria de Heidegger, que buscan prescindir, paradójicamente, de la premisa del sujeto. Si atendemos a esta indicación, un desafío que resta, pues, al desarrollo futuro de los modos de comprensión de la filosofía moral, y de la antropología filosófica, es el de cómo vincular el análisis del problema moral, de un sujeto de sentidos, con una teoría ontológica del trasfondo que es, en cambio, a-subjetiva.

Palabras clave: Taylor, fenomenología, lenguaje, razón práctica, trasfondo. 


\section{Introducción}

Podría decirse que la obra de Taylor, en su conjunto, tiene dos caras que destacan notoriamente; la primera, mira hacia el pasado de la filosofía indicando los hitos que marcan el desarrollo de una perspectiva 'equivocada' sobre la naturaleza del conocimiento y la ética. Ésta tradición es denominada como una corriente "epistemológica", o a veces, dependiendo del ámbito de conocimiento puntual, que es objeto de la crítica, como "naturalista" o "nominalista" (1). Y en segundo lugar, una cara que enfoca su perspectiva hacia una tradición de pensamiento, en el presente, que según Taylor, es fecunda para elaborar una teoría más correcta sobre todos los aspectos que abarca la especulación filosófica; en particular, una ontología completa, y una ética. En su discusión sobre el estatuto del lenguaje, Taylor denomina a esta tradición como una teoría constitutivo-expresiva. Autores importantes que la conforman son Herder, Humboldt y Hegel, como también, más actualmente, Merleau-Ponty, Wittgenstein y Heidegger.

La idea que Taylor desarrolla es -en síntesis- que en función de esclarecer los aspectos más profundos (desde el punto de vista antropológico) del estudio de la ética, existe cierta compatibilidad entre, por un lado, la noción del lenguaje que viene expuesta por la teoría expresiva de Herder, Humboldt y más recientemente Wittgenstein, y un tipo de análisis fenomenológico y hermenéutico, por otra parte, tal como es desarrollado por Heidegger, Gadamer y Merleau-Ponty. La interpretación de la historia de la filosofía que subyace a esta opinión, y el reordenamiento que Taylor propone de los respectivos acercamientos analíticos de estas dos vertientes, tiene como fundamento dos ideas centrales, que son: el concepto de trasfondo y el análisis de la situación encarnada del sujeto humano como condición ontológica primordial. En este trabajo, estudiamos el modo como Taylor entiende que la comprensión adecuada de estos conceptos permite refutar las posturas "naturalistas".

\section{El sesgo esencial de la perspectiva desvinculada}

En su artículo "Lichtung or Lebensform: Parallels between Heidegger and Wittgenstein" (1995), Taylor sostiene que un aspecto en común que destaca a estos dos pensadores es su oposición a lo que él denomina como una "ontologización" de la perspectiva desvinculada. Más aun, no es sólo que ambos se ocuparan, cada uno a su modo, de mostrar que esta perspectiva está equivocada, y que una correcta comprensión del ser humano, en cambio, ha de concebirlo a éste como un agente encarnado, sino que, además desarrollan, de distintas maneras, una forma de articular el trasfondo que subyace a esa encarnación, o incardinación del hombre en una forma de vida particular; en un cuerpo, en una cultura, y en un lenguaje.

El antecedente fundamental de la denominada "ontologización", es la afirmación gradual, en la modernidad, de un enfoque atomista para comprender el modo en que el hombre conoce, y la respectiva convicción de que la acción apropiada de la inteligencia humana radica en la experiencia del procedimiento racional, más que en la razón, en sentido sustantivo (esto es, como un medio de acceso a la realidad del mundo). Según Descartes, el hombre sólo debe atender al modo en que opera su pensamiento lógico, o matemático y no confiar en otro tipo de ideas que no tienen esta claridad y distinción, como pudieran ser, por ejemplo, los razonamientos construidos sobre la base de una experiencia "encarnada" fundada en el tipo de impresión de las "cualidades secundarias". Sólo abstrayéndonos de éstas conocemos lo que es "objetivo", en la realidad del mundo. Dice Taylor: "la concepción moderna, empezando con Descartes, se centra en los procedimientos. La razón no es aquella facultad que nos conecta a un orden de cosas en el universo, que en sí mismo pueda denominarse racional. Más bien la razón es la facultad por la cual propiamente pensamos. (...) La racionalidad supone un cuidadoso escrutinio por parte del pensamiento de sus propios procesos, lo cual determina el giro reflexivo del moderno racionalismo. Una construcción cuidadosa de nuestra imagen de las cosas requiere que identifiquemos y sigamos un procedimiento fidedigno" (Taylor 1997:95). Más adelante agrega lo siguiente respecto del requisito de objetividad: "Nuestro pensamiento es objetivo cuando escapa a las distorsiones y perspectivas de nuestro tipo de subjetividad y capta el mundo tal como es" (1997:96), es decir, que conseguimos esta objetividad abstrayéndonos del error y la confusión que supone el abandono en el sentido común de lo que percibimos en nuestra experiencia cotidiana. 
El rechazo de las ideas innatas, por parte de Locke, permite luego adaptar este racionalismo al contexto de una epistemología empirista y así provee de una explicación filosófica para las ciencias modernas, sobre todo reforzada después con Hume. En este nuevo marco, Locke provee una idea que refuerza el ideal de la desvinculación, y del sujeto de control, al postular que podemos descomponer nuestros pensamientos en una suma compuesta de ideas que representan, como pequeños átomos, o bits de información, los datos reales del mundo. Dice Taylor: "Esta concepción nos proporciona una imagen de los agentes que, al percibir el mundo, reciben bits de información de su entorno y luego los 'procesan' de cierta manera para que emerja el 'retrato' del mundo que tienen; y posteriormente estos agentes actúan sobre la base de este retrato con el fin de realizar sus objetivos, a través de un 'cálculo' de medios y fines" (1997:94). La idea resultante, es lo que Taylor denomina el "yo puntual", esto es; la de un yo que ejerce "un autocontrol reflexivo en nombre de un procedimiento canónico" (1997:95), que es el procedimiento racional.

Taylor explica que la "ontologización del procedimiento racional", o de la "perspectiva desvinculada", no se produce sólo porque creamos que para pensar acertadamente debemos separar los componentes del pensamiento, sino más bien, en un sentido más hondo, porque llegamos realmente a creer que la información entra en la mente bajo la forma de bits atómicos. La ontologización es, por consiguiente, el paso decisivo que conduce a la "interpretación del método ideal como la auténtica constitución de la mente". Como lo aclara Taylor: "Una cosa es Ilamarnos a analizar nuestras creencias en sus posibles componentes separables y otra pensar que la información primitiva que entra en la mente debe hacerlo en bits atómicos. Las 'ideas simples' de Locke son el ejemplo clásico de una semejante reificación del procedimiento llenándolo, por así decirlo, de hormigón teórico e incorporándolo en la constitución propia de la mente" (1997:95).

En suma, la raíz del proceso de ontologización es que realmente atribuimos a la constitución de la propia mente, la perspectiva desvinculada. Es decir, que esta perspectiva representa la auténtica naturaleza de la mente. Los input de información son neutros. Esto es, son un mero registro de los hechos, desprovistos de una carga evaluativa por el sujeto, y de este modo, no podemos deducir a partir de ellos ninguna entidad real. Taylor menciona dos posturas epistemológicas, en nuestra época, que continúan esta tradición del pensamiento desvinculado. Una es el dualismo cartesiano, y la otra, el monismo mecanicista. Ambas, "tanto el dualismo como el mecanicismo son ontologías de desvinculación" (1997:99). Incluso, en el marco de las actuales discusiones filosóficas sobre el funcionamiento de la mente, tal vez podríamos identificar la herencia del empirismo con las postulados conductistas, así como al racionalismo -que subyace en el dualismo cartesiano-, con las posturas funcionalistas, tanto en la vertiente de la psicología cognitiva, como en las investigaciones sobre la inteligencia artificial (2).

Esta reificación de la mente, o del procedimiento racional, como acción desvinculada, ha tenido gran influencia no sólo en el ámbito de las ciencias humanas, sobre todo en la cultura anglosajona, sino también en nuestros modos cotidianos de entender -popularmente- la mecánica del pensamiento. Esto es, de hecho, lo que ha permitido aquella facilidad con la que aceptamos -y nos hemos familiarizado con-el lenguaje atomístico-computacional, como modelo análogo del funcionamiento mental humano. El combate que llevan a cabo, tanto Heidegger, como Wittgenstein, está dirigido -dice Taylor- a refutar este modelo del sujeto desvinculado, y a proponer cada uno a su modo, una forma que explica la manera, en cambio, en que el sujeto humano se halla, necesariamente, en una condición encarnada. Taylor menciona el lenguaje, el cuerpo, y la cultura, como instancias centrales en que comprobamos esta encarnación, y además de los autores mencionados, destaca la obra de Merleau-Ponty, en especial, en relación a poner al descubierto la relación encarnada del cuerpo.

\section{La situación encarnada del hombre}

Veamos ahora en qué consisten, en general, las proposiciones de estos autores que reivindica Taylor. Una primera formulación puede dar cuenta de la siguiente evidencia. Si hemos de ser rigurosos acerca de la constitución de un mundo, o de la forma del mundo que hallamos en nuestra experiencia, vemos que es "un mundo", propiamente, en cuanto está conformado por el particular modo en que los seres humanos nos encontramos encarnados. Dicho de otra manera, nuestro mundo se conforma por nuestra encarnación "en el sentido de que el modo de experimentar o vivir el mundo es esencialmente el de un agente con su particular tipo de cuerpo" (Taylor 1997:92). Por ejemplo, 
nos orientamos en el espacio, equilibrándonos verticalmente, de forma que siempre encontramos como algo constitutivo una diferencia entre arriba y abajo aunque nos hallemos eventualmente sin ningún sistema de referencia fijo que defina estas colocaciones, en lugares, o sentidos específicos. Asimismo, encontramos que hay objetos que están ubicados "a la mano", y otros que están sencillamente fuera de alcance. Estas descripciones de la estructura, o de la forma de la experiencia, no son meramente contingentes, sino que constituyen condiciones de inteligibilidad del tipo de experiencia que es la experiencia humana. Por ejemplo, la diferencia arriba-abajo podría tal vez no aportar una descripción inteligible para otro tipo de seres que no se orienten en el espacio, o que no tengan ninguna relación ontológica con éste. Dicha diferencia no sería significativa, en ningún sentido, para ellos.

De esta manera, decimos que la encarnación corporal sirve como un trasfondo de comprensión de nuestra experiencia, sin el cual, en efecto, ésta no se puede entender adecuadamente, o bien, perdemos de vista su núcleo constitutivo. Respecto de este trasfondo dice Taylor: "No es aquello de lo que soy simplemente inconsciente (en el mismo sentido en que no soy consciente de lo que ocurre en la cara oculta de la luna) puesto que es lo que hace inteligible aquello de lo que soy indiscutiblemente consciente. Al mismo tiempo, no soy explícita o focalmente consciente de ello, ya que esta posición está ya ocupada por lo que está haciendo inteligible" (1997:101).

La obra de Heidegger y Wittgenstein, como la de Merleau-Ponty no se limita, sin embargo, a desvelar esta condición del trasfondo, y su necesidad ontológica, sino que propone además, cada una desde distinto ángulo, una particular forma de articulación de ese trasfondo. El caso de Heidegger es emblemático pues es sabido que tanto en la primera etapa de su pensamiento, como en la segunda, después del giro de 1949 (Die Kehre), la perspectiva del ser, y el estado en que éste se realiza (3), es siempre descrito como una situación encarnada.

En Ser y tiempo, puntualmente, -en el marco de lo que Heidegger denomina la "analítica existenciaria" del ser ahí (da-sein)-, encontramos esta fundamentación del ser humano como sujeto encarnado, aunque aquí, por supuesto, la encarnación no se refiere a la situación corporal -o espacial- de un individuo humano, sino más bien a la condición ontológica del ser-ahí, de tal modo que antes que "encarnado" en este caso parece mejor decir "situado", o mejor todavía, "constituido". Incluso la palabra sujeto no se corresponde con la filosofía heideggeriana que precisamente busca romper con la metafísica moderna del "sujeto". A lo que se refiere Taylor, probablemente, en cuanto reconoce igualmente, en Heidegger, la idea de la encarnación, ha de ser el hecho que la situación ontológica del ser-ahí supone de antemano que el ser-ahí es ya un ser en el mundo; que el ser en un mundo, es algo constitutivo del modo de ser del ser-ahí, y de esta manera no puede pensarse como algo desvinculado del trasfondo. Podemos ver esto en el aparato conceptual que Heidegger nos propone. Lo que él define, de hecho, como existenciariedad, corresponde al complejo de las "estructuras ontológicas de la existencia" (Heidegger 1968:22). Es decir, se refiere a un marco de condiciones que no son contingentes, o subjetivas, sino que son inherentes a esta forma de vida que es el ser humano, y que son, por tanto, condiciones existenciales desde el punto de vista de este ente. Entre tales estructuras ontológicas, inherentes al ser ahí, está precisamente -entre otras- el "ser en el mundo" (In-der-Welt-sein). Pero esto nunca se reduce a una cuestión espacial. No quiere decir -fundamentalmente- que el hombre está en el mundo, en un sentido accidental, o contingente, de la misma forma que, por ejemplo, el agua "está" en el vaso, o la mesa "está" en la habitación, sino más bien, en un sentido más profundo, que este ser en el mundo, "determina en forma radical su 'estricto hecho de ser'" (Steiner 1999:160) (4). Desde este punto de vista, por tanto, es obvio que la perspectiva desvinculada pierde todo crédito.

Taylor parece aludir a esta propiedad, en particular, -de hecho- cuando concibe que Heidegger refuta la postura del enfoque desvinculado. Acerca de esto dice: "En Sein und Zeit Heidegger sostiene que las cosas se descubren primero como parte de un mundo, es decir, como los correlatos de un compromiso relativo y dentro de la totalidad de tales compromisos" (Taylor 1997:106). Esto cuestiona el atomismo de input, a la vez, que disminuye la plausibilidad de la hipótesis de la supuesta neutralidad de los hechos (5), a través de mostrar que las cosas que descubrimos, en la naturaleza, "se descubren en un mundo a mano" (1997:106). Lo que quiere evidenciar Taylor por esta vía es la lectura de Heidegger según la cual es un error interpretar nuestra percepción de las cosas fundamentalmente como algo neutral. El concepto de "ser a la mano" (Zuhandenheit), derivado del hecho de nuestro "ser en el mundo" como condición existenciaria, pone al descubierto que esto no es así, y que, por el contrario, nuestra fundamental relación 
con los objetos, no es la que nos revela su forma "ante los ojos", característica de nuestra actitud teórica, y especulativa, sino de un modo mucho más esencial y arraigado en el verdadero fenómeno -o experiencia-, por la manera en que los vivenciamos, o los utilizamos como parte de nuestra vida, y cuyo sentido "vivido" queda mucho mejor descrito con el término "a la mano". Dice Taylor: "El objetivo es mostrar que captar las cosas como objetos neutrales es una de nuestras posibilidades sólo a partir del trasfondo de un modo de ser en un mundo en el que las cosas aparecen como a mano. Captar las cosas de modo neutral requiere modificar nuestra actitud hacia ellas, la cual originalmente es la de estar vinculado" (1997:107). Esto equivale a decir que la condición encarnada es lo verdaderamente esencial, y lo que da cuenta de nuestra verdadera naturaleza humana. Esto nos demuestra, en suma, "que no podemos atribuirle a la postura neutral el lugar paradigmático en nuestras vidas que la imagen desvinculada le atribuye" (1997:107).

En su libro "Fenomenología de la percepción”, Merleau Ponty provee un conjunto vasto de reflexiones, amparadas en antecedentes tomados de la psicología, que ratifican esta perspectiva a favor de la explicación encarnada, en relación -esta vez sí- con el cuerpo y su espacialidad. La prerrogativa ontológica del ser en el mundo frente al mero hecho de la objetividad neutral de las cosas, es suscrita por Merleau Ponty en relación con la realidad del cuerpo, bajo la idea de que éste no es un simple objeto del mundo, entre otros que hallamos, ya dispuestos, sino que es, más bien, en un sentido primordial, nuestro medio de comunicación con ese mundo en sí mismo, donde aparecen esos objetos. Dice el pensador: "La permanencia de mi cuerpo no sólo no es un caso particular de la permanencia en el mundo de los objetos exteriores, sino que la segunda únicamente se comprende por la primera; la perspectiva de mi cuerpo no sólo no es un caso particular de los objetos, sino que aun la presentación en perspectiva de los objetos sólo se comprende por la resistencia de mi cuerpo a sufrir una variación de perspectiva" (Merleau Ponty 1957:91). Aquí la encarnación es de nuevo lo fundamental y la piedra de toque, como base ontológica. El autor francés ataca, de hecho, las concepciones tradicionales de la psicología clásica puesto que éstas, al desestimar el fenómeno de la experiencia subjetiva del cuerpo, bajo la exigencia de observar y centrarse únicamente en las propiedades absolutas del objeto como tal -desde una perspectiva desvinculada-, convierten esa "experiencia del cuerpo" en una mera "representación del cuerpo" que tiene lugar en la dimensión de lo "psíquico". Esto, lo psíquico, es un ámbito que se opone a lo real, pero que, en vez de requerir una nueva definición del ser, se asume que es susceptible de someter al análisis estrictamente científico -positivo-, del mismo modo que al cuerpo. De esta manera, esa experiencia, que exigía un tratamiento fenomenológico, para aproximarnos, en verdad, a su comprensión, se reduce a un "hecho psíquico" (Merleau Ponty 1957:101) (6) que, aun cuando no podemos explicar todavía del todo, ya lo hará una vez que las ciencias positivas consigan el avance requerido.

\section{La deducción trascendental de Kant y el trasfondo}

El enfoque desvinculado plantea que las ideas están conformadas como bit de información, dotadas de sentido por sí mismas, y de esta manera, aunque no lo explicite, este tipo de perspectiva tiende a ocluir la condición del trasfondo. La idea de que el conocimiento de la realidad nos llega en bits particulares, o en impresiones individuales, es desarrollada por Locke y luego por Hume. En la perspectiva de Hume, estas impresiones atómicas son asociadas, en un estadio posterior de elaboración del pensamiento, mediante el cual afirmamos psicológicamente ciertas creencias como, por ejemplo, la de la relación causa-efecto. No obstante, nuestro análisis introspectivo puede siempre descomponer estas conclusiones y revelar así el original estado básico de las impresiones separadas. Es por esta vía, de hecho, que nos damos cuenta de que no existe, en realidad, esa necesaria conexión entre la causa y el efecto (Taylor 1997:104). Lo cierto son las impresiones sensibles particulares, pero no esas creencias construidas por asociación.

Los argumentos dados por Kant, en el marco de la deducción trascendental, se pueden considerar, no obstante, como un primer estadio en la reposición de la idea del trasfondo. Lo que Kant cuestiona es, precisamente, la premisa de que las impresiones particulares, consideradas al modo de Hume (como bits atómicos de información), pueden, en realidad, tener por sí mismas, (independientemente de todo correlato, o trasfondo), un significado acerca de algo. Por el contrario, la "intencionalidad", o el "ser acerca de" algo, es una condición necesaria -en primer lugar- para que cualquier impresión sensible pueda significar algo real, y esto es una circunstancia que reconoce también la 
postura empirista, para el caso de los objetos externos que corresponden a este tipo de impresiones (7). Dice Taylor: "Kant habla" a este respecto "de la necesaria relación entre el conocimiento y su objeto". Pero Kant no se detiene en este punto, y va más allá, pues muestra que esta relación no sería posible, de ningún modo, "si tuviéramos que tomar" la impresión de modo aislado, sin ningún vínculo con otras impresiones, u objetos (Taylor 1997:105). A lo que se refiere Taylor, en el fondo, es al principio de la unidad objetiva de la conciencia, que, como se sabe requiere necesariamente-dentro del marco explicativo del sistema trascendental- de la síntesis de las categorías. Dice Taylor, aludiendo a este principio: "La unidad de este mundo es presupuesta para todo lo que se presente por sí mismo como un bit particular de información y, por lo tanto, sea lo que fuere lo que queramos decir con tal bit, no puede ser totalmente independiente en relación con los demás. (...) Conseguir, de hecho, romper todos los vínculos entre las impresiones individuales sería perder cualquier sentido de la conciencia de algo" (1997:105).

A esto se refiere Taylor con esa necesaria "unidad del mundo", que debe ser presupuesta para el sentido posible de los bits individuales, y que es lo que destruye, precisamente, la opción de que éstos alcancen ese significado por sí mismos, como postula Hume. Es así que dice Taylor: "La deducción trascendental, junto con los argumentos correspondientes en la Crítica de la razón pura, puede considerarse como un cambio decisivo en la filosofía moderna. Retrospectivamente cabe concebirla como el primer intento de articular el trasfondo requerido por las operaciones que el propio retrato desvinculado describe como inteligibles" (1997:105).

La perspectiva desvinculada, de hecho, tiende a ocluir, o a invisibilizar este trasfondo, pues presupone, equivocadamente, que "las condiciones de inteligibilidad están incorporadas en los elementos y procesos de la mente en tanto que propiedades internas". Es decir, considera que "la impresión aislada es información inteligible por sí misma", que "tienen la entera existencia particular y separable de un objeto externo" (1997:105). Este punto de vista -concluye Taylor- olvida que, para que algo sea inteligiblemente $X$, tiene que contar como inteligible $X$, y siempre hay condiciones contextuales para que cualquier cosa cuente como algo" (1997:105). Estas condiciones contextuales constituyen el trasfondo.

\section{La teoría constitutivo-expresiva del lenguaje}

Taylor considera que existen dos dimensiones distintas de comprensión del significado, las que designa como teoría designativa, o nominativa, representada por la tradición que personifican Hobbes, Locke y Condillac, y la teoría expresiva, o expresivo constitutiva -como la llama en su artículo Heidegger, Language and Ecology (Taylor 1995)-, y cuyo desarrollo encontramos primero en la obra de Herder, y más tarde profundizada en Humboldt, Sausurre, Heidegger y Wittgenstein.

La primera de estas dimensiones, o concepciones del lenguaje, la teoría nominalista, tiene sus raíces en la epistemología moderna, que entiende al conocimiento humano de modo atomista y representacional. Es una epistemología que comienza con las ideas de Descartes, pero que incluso tiene antecedentes en etapas más tempranas, en el pensamiento ockamista. Locke es una figura importante en la conformación más definitiva de esta postura filosófica, y en la manera en que esta concepción del lenguaje queda vinculada con una particular teoría de la mente. Según el modelo representacionalista, el conocimiento de las cosas se produce por medio de las ideas, las que copian, o reproducen, en el interior del sujeto (8), una presentación de estas cosas externas, o bien del ensamblaje entre ellas, de modo tal que el pensamiento correcto -el "conocimiento" propiamente tal- se realiza cuando este ensamblaje interno de las ideas se corresponde, efectivamente, con la forma como están conectadas las cosas en la realidad objetiva. Las palabras adquieren, pues, su significado, precisamente, en conexión con estas ideas. El pensamiento consiste, en suma, en "ensamblar ideas; más específicamente, en el ensamblar ideas claras y distintas según el modo en que están ensamblados los elementos constitutivos del mundo" (Taylor 1985:225) (9).

Es importante ver que, en este contexto, el lenguaje no puede ser más que un mero instrumento anexo, para realizar, y controlar, este ensamblaje de las ideas. El significado de una palabra ha de consistir sólo en la cosa que designa, la que es representada como un bits en nuestra mente, y no se puede ir más allá, y pretender para ella una realidad correspondiente que el hombre descubra, digamos, por medio de la razón. Además, para esta teoría, la asociación de ideas que constituye el pensamiento, es un contenido anterior, e independiente del lenguaje, mientras que lo que 
añade el uso lingüístico, propiamente, es el mero control al que podemos someter estos pensamientos. El lenguaje es concebido, por tanto, como una herramienta que facilita la tarea de ensamblar nuestras ideas de acuerdo al ensamblaje que prevalece en la realidad "exterior". Para ello debe ser lo más transparente posible, y no dejar espacio para ninguna ambigüedad respecto de la definición correcta, o el campo semántico, de una palabra, y su uso coherente. De esta manera, lo que se busca es obtener el mayor control que nos sea accesible, respecto de la construcción de nuestro propio léxico, evitando así la pérdida de libertad, que significaría, de otro modo, que el lenguaje por su cuenta plasmara nuestro pensamiento. Como dice Condillac, el lenguaje nos proporciona el control sobre el proceso de asociación.

Una consecuencia lógica que, en particular, se puede derivar de la propuesta de Locke, y que suscita controversia, es que si la palabra adquiere su significado por medio de la idea, parece que el lenguaje fuese, en lo fundamental, una cuestión privada. Las palabras usadas podrían corresponderse con las mismas cosas que enuncian, para todos los individuos, pero el significado de ellas, al estar ligado a las ideas internas, sería diferente, pues variaría según los sujetos. Una de las cosas que intentó demostrar Wittgenstein era precisamente la imposibilidad de esta hipótesis de un lenguaje privado (10). Para ello, esgrimió el criterio de la corrección intrínseca de las palabras, lo que significa que el significado de éstas -o en general, de los signos-, no se obtiene a partir de su adecuación a ninguna condición extrínseca al lenguaje mismo. Esta explicación reafirma los postulados que Herder ya había expresado, acerca de la reflexividad del ser humano, como premisa ontológica del lenguaje, o, en la denominación de Taylor, la idea de que el hecho crucial del significado es la participación del hombre en una "dimensión semántica".

Taylor identifica el concepto del trasfondo con esta participación del hombre en la "dimensión semántica", y en este sentido, constituye el postulado central de la teoría constitutivo-expresiva. La dimensión semántica es un ámbito en que los signos -o las palabras- son formas de significado que, en sí mismas, son irreductibles a cualquier criterio que sea ajeno al lenguaje mismo. La existencia de esta dimensión equivale, pues, a decir que hay un trasfondo de significación que es, en realidad, lo que hace posible el lenguaje. Lo que muestra Wittgenstein es, precisamente, que el tipo de definición ostensiva sólo es posible si el lenguaje, por decir así, pre-existe, lo que puede verificarse, por ejemplo, en la forma de una pre-comprensión de sus operaciones internas, por parte del sujeto, y del lugar que ocupa la palabra dentro del conjunto dinámico articulado bajo la forma de unos "juegos de lenguaje". Esto rechazaba rotundamente la postura en que los nominalistas fundaban su teoría, es decir, que las palabras adquieren su significado simplemente al designar los objetos, en el uso regular que se hace de ellas.

\section{La encarnación del lenguaje, en las formas de vida}

La idea de que el significado de las palabras presupone el lenguaje como un todo es una propiedad que Taylor identifica como un cierto "holismo del significado". El precursor de este descubrimiento es Herder. Así, dice Taylor: "Una de las consecuencias más importantes y universalmente reconocidas del descubrimiento herderiano es un cierto holismo del significado. Una palabra sólo tiene significado dentro de un léxico y de un contexto de usos de lenguaje que, en último término, están incrustadas en un modo de vida" (1997:133). Los razonamientos de Wittgenstein continúan este postulado, que señala la existencia de un trasfondo, y ratifican también, de hecho, la noción del lenguaje como una realidad situada, o encarnada en las formas de vida (Lebensform).

Un componente esencial de esta perspectiva es la idea de que el pensamiento y la palabra son, en el fondo, la misma cosa, idea que Herder había tomado originalmente de Hamann. Es decir, no es que los hombres primero piensen, mediante conceptos o ideas, "para luego buscar las palabras" con que "vestir", o envolver estos pensamientos, de la misma forma en que, por ejemplo, buscamos un guante que se ajuste a nuestra mano (Berlin 2000:214), sino que más bien, debiésemos entender que el lenguaje es constitutivo del pensamiento, y que no pensamos, en rigor, si no es con las palabras, y sus significados intrínsecos. El meollo de este postulado está en la hipótesis de la naturaleza reflexiva del hombre, y que, como vimos, es la base de la teoría expresiva. Se trata, entonces, de una cuestión antropológica, de plano. Esto es, que el sujeto humano es, por esencia, un ser que, a diferencia del resto de los animales, participa -por su reflexión- de una dimensión semántica -o de significados- que hace posible el pensamiento. En cuanto somos reflexivos, o en cuanto reflexionamos, -o actualizamos nuestra postura reflexiva(11), participamos de esta dimensión semántica, de modo que el acto del habla viene a ser la expresión de esta 
participación en el pensamiento. Taylor explica esto refiriéndose en los siguientes términos a la postura reflexiva: "La acción que expresa y actualiza esta nueva postura es el habla. El habla es la expresión del pensamiento. Pero no es simplemente un ropaje exterior de lo que podría existir independientemente; es constitutivo del pensamiento (lingüístico) reflexivo, del pensamiento que tiene que ver con sus objetos en la dimensión lingüística" (1997:132).

En último término, dado que nuestros pensamientos expresan nuestros sentimientos, nuestras creencias, y actitudes, y en general, nuestras formas de vivir, o percibir el mundo, resulta entonces que el lenguaje -que es la articulación de ese pensamiento- está situado, o encarnado en estas formas de vida. Dice Taylor, "nuestra capacidad para funcionar en la dimensión lingüística está atada, en sus usos cotidianos, así como en sus orígenes, al habla expresiva, como la gama de acciones en las que no sólo es comunicada sino realizada" (1997:132). Es decir, que el lenguaje expresa lo que somos, y el pensamiento se articula sobre la base de esta expresión. La lengua no es solo una herramienta para comunicarnos, sino que es constitutiva de un modo de estar en el mundo, y transmite -o porta-, en este sentido, una cierta identidad. En palabras de Benedicto Rodríguez: “Cada acto de habla tiene como trasfondo la totalidad de un lenguaje que expresa un modo de vida" (Benedicto Rodríguez 2004:178).

Pero esta singular circunstancia del lenguaje, como un trasfondo que revela un todo de una forma de vida, implica también que el lenguaje no puede ser un acto particular, de los individuos aislados. El lenguaje no es susceptible de ser controlado, o dominado por los individuos: "No puede ser nunca sólo mi lenguaje, sino que es siempre, en gran medida, nuestro lenguaje" (Taylor 1985:234) (12). Y esto tiene una importante repercusión en el sustrato que imaginamos de nuestras formas sociales. En su estudio sobre Herder, Berlín evoca esta implicancia. Dice: “Los grupos humanos (...) se convierten en una unidad a partir de tradiciones humanas comunes y memorias compartidas cuyo principal enlace y vehículo -de hecho, más que un vehículo, su encarnación misma- es el lenguaje” (Berlin 2000:214). La comunidad, en suma, es una instancia constituyente del lenguaje.

La situación del trasfondo desacredita, así, la concepción antigua del lenguaje, que, de modo atomista, "sitúa al pensamiento y el conocimiento en la mente del individuo" (Taylor 1997:110) y plantea la posibilidad de un lenguaje privado. Si volvemos, en efecto, la mirada a la situación específica de los sujetos humanos con el lenguaje, y sus relaciones de comunicación, la hipótesis del trasfondo equivale a sostener que el lenguaje, fundamentalmente, "viene modelado y crece ante todo no en el monólogo, sino en el diálogo, o mejor en la vida de la comunidad hablante" (Taylor 1985:234) (13). El contraste en este punto, entre la concepción nominalista y la expresiva, no puede ser más extremo. El enfoque nominalista, centrado en una concepción atomista y desvinculada de la situación humana, considera que el conocimiento se realiza en inputs que procesan la información al interior de las mentes. Pero este proceso no puede tener otro lugar más que en los individuos tomados de modo particular. Esta idea se refuerza aún más, dice Taylor, bajo una explicación mecanicista, según la cual el pensamiento se realiza "dentro" de organismos individuales, puntualmente, en el cerebro. Desde esta perspectiva, la manera como compartimos el lenguaje viene a ser nada más que por convergencia. "Lo cual significa, por ejemplo, que mi lenguaje es cercano al tuyo y al suyo" [de este modo] "si he de ser un hablante, entonces ha de haber algo así como mi lenguaje, mi idiolecto; y si ha de haber algo como el conocimiento común, primero ha de haber mi conocimiento, el tuyo y el suyo" (1997:110).

\section{El lenguaje y la razón práctica}

La concepción meramente instrumental del lenguaje, que prima en la teoría nominalista, considera que podemos definir los pensamientos del hombre $-y$, por tanto, las razones que subyacen a sus propósitos y a la determinación consciente de su voluntad-, al margen del lenguaje. Como acabamos de ver, no obstante, esto no es posible, enfocado desde la perspectiva expresiva, que concibe al lenguaje como algo constitutivo del pensamiento mismo. En este caso, los razonamientos, o juicios, que fundan la facultad de agencia humana, más que revestirse, o utilizar las palabras, o signos para realizarse, como si fueran sólo su medio, o instrumento ad hoc, expresan ellos mismos, activamente, las posibilidades de unas formas de vida, que están encarnadas en el lenguaje. 
Para entender del todo, no obstante, cómo se vinculan el lenguaje y la razón práctica, en este sentido, bajo la perspectiva del enfoque "constitutivo-expresivo", es necesario atender al hecho que el rasgo peculiar del hombre es, precisamente -como dijimos-, que participa dentro de una "dimensión semántica". El uso humano del lenguaje supone, en efecto, la entrada en una dimensión de significación en que las palabras poseen un sentido intrínseco de corrección. Y esto implica establecer una diferencia importante entre el lenguaje humano, propiamente tal, y el mero signo, pues este último puede ser descrito como una mera tendencia a realizar sólo unos objetivos extrínsecos al lenguaje, o a satisfacer ciertos objetivos operacionales, en un sentido que es, efectivamente, sólo "instrumental". En su artículo "La importancia de Herder" (Taylor 1997), Taylor ilustra este punto con la diferencia entre la capacidad de operar con signos, de los seres prelinguísticos, y el uso del lenguaje propiamente tal. Pongamos el caso -dice- de un chimpancé que aprende a señalar el casillero correcto que representa a la "banana", porque con ello consigue la fruta, o el de una rata que cruza correctamente la puerta con el triángulo y no con el cuadrado, porque con ello consigue el queso. En ambos casos, "aprender a usar el signo es aprender a aplicarlo apropiadamente para la satisfacción de algún propósito o tarea no-lingüísticamente-definidos" (Taylor 1997:121). El criterio es siempre extrínseco al lenguaje. La relación -dice Taylor-va del éxito al signo, y esto define la corrección. Pero la situación es muy distinta, en cambio, en el caso de algunos usos del lenguaje humano como, por ejemplo, en "la descripción científica desinteresada, la expresión de los propios sentimientos, la evocación de una escena en verso". Ciertamente, podemos decir que una metáfora es correcta, o profunda, y aquí hay una cierta "corrección" implícita, pero en este caso, como asimismo en los casos puntuales que podamos imaginar respecto del resto de las situaciones mencionadas, esta corrección no se puede explicar "en términos de una tarea que no esté, en sí misma, definida lingüísticamente". Esto significa que aquí los criterios son intrínsecos al lenguaje mismo. Es decir, que "no podemos definir la exactitud de la palabra a través de la tarea sin definir la tarea en términos de la corrección de las palabras. No hay ninguna relación unidireccional que pueda traducir la corrección de la palabra en términos de alguna forma de éxito, definida independientemente. La exactitud no puede ser explicada de forma reductiva" (Taylor 1997:121).

Esto es, en definitiva, lo que busca expresar Taylor al decir que en el caso de estas actividades estamos frente a unas formas irreductibles de corrección de los signos, y que, por consiguiente, las criaturas que participan de este tipo de corrección o significación, son parte de la denominada "dimensión semántica". Una criatura, como el hombre, que participa de esta manera, en el ámbito del lenguaje, es un ser que es "sensible", en este sentido, "a cuestiones irreductibles de corrección" (Taylor 1997:122). Y esta es, en definitiva, la principal diferencia, con la noción fundamental incorporada en la teoría nominalista del significado, que se centra únicamente en las relaciones objetivas, u objetivables materialmente, para el cumplimiento de una función establecida de modo extrínseco al lenguaje mismo, y que deja de lado, por tanto, la realidad expresiva de las palabras, y del lenguaje mismo, en general.

Estas apreciaciones -en su conjunto- pueden enfocarse en relación con la respectiva concepción de la persona humana, que expone Taylor, a lo largo de sus escritos, y que se opone al concepto que de esto mismo tiene el naturalismo. Los seres humanos, como piensa Taylor, somos, en lo fundamental, sujetos que no actuamos sólo para satisfacer nuestros deseos de facto, sino que somos capaces de valorar estos deseos y el conjunto de nuestras propias inclinaciones, a la luz de criterios de significancia que están vinculados al tipo de persona que queremos ser. En esta esfera de nuestra acción, el lenguaje cumple un rol fundamental que ahora, una vez que ya hemos consignado los principales rasgos ontológicos del lenguaje, y en particular las propiedades suyas que acabamos de mencionar (identificadas desde el ángulo de la teoría constitutivo-expresiva) podemos entender.

\section{El hombre como animal que se auto-interpreta}

En varios de sus trabajos Taylor sostiene que la condición del agente humano es, en particular, la de un sujeto que prueba emociones que no responden sólo a constituir la reacción apropiada para ciertas situaciones. Esto significa que, para el ser humano, los eventos no comportan unos meros hechos brutos, o ante los cuales realizamos un mero cálculo de acciones con miras a conseguir un objetivo puramente operacional. Existe un tipo de emociones, que son propia y exclusivamente humanas -como la vergüenza, o el orgullo- o que son "relativas al sujeto" -o, más precisamente, que poseen un "import" relativo al sujeto-, en el sentido que tienen para éste una relevancia que los hace significativos según el ideal, o modelo de vida, al que aspiramos. En su artículo titulado Self-Interpreting Animals 
(1985), Taylor introduce específicamente este concepto y aclara la respectiva peculiaridad de los import "relativos al sujeto". Los import, en general -explica- son propiedades objetivas de las cosas, o de las situaciones en que estamos involucrados en nuestra vida, como agentes prácticos, y que tienen relevancia para nosotros en relación a nuestro ideal práctico. Literalmente dice que el import-que podríamos traducir aquí por "relevancia"- se refiere "al modo en que algo puede ser significativo o relevante para los deseos o los objetivos o las aspiraciones o los sentimientos de un sujeto" (Taylor 1985:48) (14). Ahora bien, hay algunos imports como la amenaza (que suscita la emoción del miedo) que -tal como quiere el naturalismo- bien podrían explicarse en los términos de meras relaciones objetivas. Una situación amenazante, por ejemplo, podría ser definida, perfectamente, como una situación que, en virtud de mecanismos causales, tiene una cierta probabilidad de provocarnos algún perjuicio concreto en nuestro estado, o integridad física. Así -dice Taylor-, es muy probable que un león hambriento nos ataque, y que podríamos incluso imaginar una máquina que sepa reconocer a un león, identificar, luego, en un banco de datos, los hechos relativos a un león, y, en consecuencia, el peligro asociado; y enviar, posteriormente, las instrucciones correspondientes al mecanismo de locomoción para activar la huída. En un caso como éste, tendríamos, según parece, "un perfecto equivalente estructural del miedo, que incorpora incluso el reconocimiento del import del peligro y la fuga" (Taylor 1985:52) (15), es decir, de la amenaza, sin la necesidad de hacer ninguna alusión adicional a la experiencia subjetiva.

Pero algo muy distinto ocurre, sin embargo, en el caso de otros import, en los que esta reducción de la explicación naturalista ya no resulta posible. Taylor coloca el ejemplo de la situación -o el objeto moral- que activa la emoción de la vergüenza. Aquí la situación no puede ser explicada en términos de relaciones meramente objetivas, pues la propiedad involucrada que activa esta emoción sólo es un import para un sujeto para quien las cosas pueden tener un sentido, o un significado. Para ilustrarlo, Taylor nos invita a pensar en la vergüenza que, por ejemplo, nos pudiera provocar, como hombres, el tener una voz estridente, o unas manos afeminadas. Estas propiedades sólo pueden avergonzarnos porque tienen un significado; porque son signo de algo poco viril, que hace pensar en algo "histérico", "no controlado", "no sólido", o "no fuerte". Es imposible explicar, en cambio, estas propiedades desde el punto de vista de meras relaciones entre objetos, o como algo que podría probar una máquina. Y esto obedece a que la propiedad es "vergonzosa", porque apunta estrictamente a la experiencia que tiene un sujeto acerca de un significado. Esto es, de un sujeto en "cuya forma de vida está presente una aspiración a la dignidad, a ser una presencia entre los hombres que exige respeto" (Taylor 1985:52) (16). Son estos significados específicos, que puede tener una propiedad (el import) de las situaciones, las que motivan la emoción de la vergüenza, y no una causalidad meramente objetiva (17). En términos muy simples, diríamos que lo que se requiere para la experiencia de este tipo de significados es un sujeto autoconciente, o conciente de lo que él es, o de lo que quiere ser, capacidades que no parece que podamos atribuir a una máquina. Lo que se busca subrayar, en esto, es la dimensión subjetiva irreductible. Taylor dice: "Lo vergonzoso no es, de hecho, una propiedad que puede valer para cualquier cosa independientemente de la experiencia que los sujetos hacen de ella. Más bien, la descripción misma del significado de la vergüenza comporta la referencia a cosas -como nuestro sentido de la dignidad, del valor, del modo en que somos considerados por los otros- que están esencialmente vinculadas a la vida de un sujeto de experiencia" (1985:52) (18).

Hay, por tanto, una diferencia crucial entre el import de la amenaza, y el ya mencionado import de la forma de las manos, por ejemplo, y que radica en que este último es una propiedad que Taylor llama "relativa al sujeto", para diferenciarla de los import, como el del primer tipo, que pueden carecer de esta relación esencial con un sujeto conciente, o de significados. El rasgo particular que caracteriza al ser humano como un ser que se auto-interpreta es, precisamente, que es un ser que experimenta este tipo particular de import "relativos al sujeto".

Por último, esta concepción de la persona humana, como un ser de significados, está relacionada estrechamente con la concepción del lenguaje, en el marco de la perspectiva "constitutivo-expresiva", pues Taylor señala que el modo específico como se realizan las articulaciones del sentido, o del significado, en el hombre, no puede ser otro que el lenguaje. En su libro "Fuentes del yo", Taylor llama "discriminaciones cualitativas" (19), al tipo de valoraciones fuertes que constituyen este proceso de articulación. Así discriminamos algunos de nuestros deseos, o pretensiones, como más dignos, y menos dignos, más elevados, o triviales, etc. Lo que está planteando Taylor es, en el fondo, que la verdadera fuerza de estas discriminaciones requiere del lenguaje, o bien, que el lenguaje es el vehículo que nos 
permite realizar esa articulación de sentidos, y las respectivas discriminaciones. Taylor es muy claro a este respecto, y plantea que aquello que define la "profundidad" como rasgo distintivo -por excelencia- de los seres humanos, es, de hecho, la preocupación por el sentido. Pero estas preocupaciones, exclusivas del hombre -añade Taylor-, "comparecen sólo en el lenguaje, y pueden ser sólo las preocupaciones de un animal de lenguaje" (1985:263) (20). De este modo, y con esta analogía, nuestro autor identifica, en definitiva, el sentido de profundidad, y la capacidad de dar significado existencial a su vida, por parte del hombre, con la posesión del lenguaje, que, como vimos, es de hecho, caracterizada como un habitar en una dimensión semántica, o un mundo de significados.

En suma, es sólo a través del lenguaje que el hombre adquiere conciencia de los valores que asigna a las cosas, y puede experimentar, por consiguiente, estas cosas como buenas o virtuosas. Pero este lenguaje no puede ser creado o inventado por el sujeto de manera independiente, en el espacio virtual del monólogo, sino que, como dijimos, el lenguaje es esencialmente dialógico, de tal manera que es, en cambio, el espacio común, o de interlocución entre los hombres, el que funda el horizonte, o el trasfondo moral, dentro del cual se mueven y escogen también sus opciones morales personales, los hombres.

\section{Conclusiones}

El estudio llevado a cabo en este trabajo, acerca del concepto de la razón práctica en la obra de Charles Taylor, nos ha permitido explicitar como núcleo central de sus ideas lo siguiente: el ser humano (homo sapiens), a diferencia de la máquina (machina sapiens) (21) y del animal, es un ser de significados. El carácter intencional de su modo de conciencia está definido por este rasgo peculiar, y característico suyo, producto del cual su estado normal, en la facticidad de su existencia, consiste en buscar ya de antemano, y necesariamente, determinados sentidos, o significados, para las situaciones prácticas que constituyen su vida. En particular, lo que aquí se ha tomado como aspecto fundamental de investigación, ha sido una característica específica del uso de la razón en su sentido práctico, por los seres humanos, que Taylor interpreta como un factor constitutivo de una ciencia de la praxis humana. Este antecedente ontológico fundamental es lo que Taylor, -siguiendo una tradición conceptual de la fenomenología, y la hermenéutica- denomina el trasfondo, u horizonte de pre-comprensión.

El aspecto fundamental de este concepto del trasfondo es que constituye un horizonte de referencia que es ontológicamente anterior al dominio de la razón subjetiva del agente individual. En la formulación particular de Taylor, no se trata, no obstante, de considerar sólo el trasfondo como condición de posibilidad de la experiencia intencional, o consciente (no es concebido, en lo esencial, como un requisito gnoseológico) -como en Kant-, pero tampoco se reduce a la mera pre-comprensión social, o inter-subjetiva del mundo, realizada en el lenguaje -como en Humboldt, o Herder-, aunque la incluye también a ésta. Sino que además, en base a la caracterización dada del hombre, como animal que se auto-interpreta, busca centrarse en el modo en que el horizonte, o trasfondo, es el fundamento de la deliberación personal del sujeto humano, en cuanto agente moral, o de praxis. Esto es lo que conecta el examen tayloriano al programa de investigación de la fenomenología, y apunta al tipo de exploración que Heidegger lleva a cabo en el marco de la analítica existenciaria.

Una vía alternativa que Taylor explora en sus escritos, para dar cuenta de la situación de pre-comprensión que provee el trasfondo, u horizonte, es la que lo identifica a éste con la propiedad del lenguaje, como una dimensión semántica, cuyo dominio, sobre la conformación de los sentidos, es anterior a la que introduce la intencionalidad de los sujetos particulares. Hay una diferencia ostensible, sostiene Taylor, entre la concepción representacionalista del nominalismo, respecto del lenguaje, y de la mente, en comparación con el conjunto de planteamientos que conforman lo que denomina la base de una teoría "constitutivo-expresiva". Para los nominalistas, en general, la aparición del lenguaje, o del significado, es entendida considerando la simple denotación ostensiva. Pero lo que esta descripción deja inexplicado es el modo en que una palabra toma el lugar de una cosa, y que es esto, en efecto, lo que comporta el verdadero misterio de la significación. La cuestión decisiva es, por tanto, ésta; es decir; cómo es que se une el lenguaje con el mundo. En el pensamiento de autores como Herder, Humboldt y Wittgenstein, Taylor cree encontrar una explicación satisfactoria, o al menos una vía fecunda para abordarla. En realidad, no sabemos -con precisión- como sucede eso, y la vinculación ontológica entre el mundo y el lenguaje sigue siendo un arcano. Pero sí sabemos, al menos, que quienes lo hacen -o bien, cuya experiencia intelectual refleja dicha operación- son aquellos 
sujetos, como los seres humanos, que son sujetos de sentido, y que participan por ello de una "dimensión semántica", o del "lenguaje" propiamente, mientras que aquellos que no participan de esta dimensión, no se puede decir que sean seres de sentido; en estos casos, aunque en ciertas circunstancias, sus acciones parezcan responder a directivas, o indicaciones verbales, no pueden sino considerarse más que como meras "reacciones a señales", en sentido bruto, pero no como "lenguaje".

Una distinción esencial en esta dirección que aportan las argumentaciones propias de los autores que, en su conjunto, contribuyen a una teoría constitutiva, es que dicho mundo o dimensión del sentido, que conforma el lenguaje, no puede ser un producto del mundo empírico, o un reflejo de él, como es lo que sí parecen sostener, en cambio, los razonamientos del nominalismo, o del representacionalismo empirista (en el caso de la filosofía de la mente). Wittgenstein, por ejemplo, es claro, en afirmar que la lógica-dentro de la cual tendríamos que incluir la lógica del sentido, en el lenguaje- es por completo independiente del mundo, o de la realidad empírica, pues no dice nada acerca de hechos. No obstante ello, los objetos del mundo humano -esto es, del mundo en que comparecen objetos intencionales, articulados con un significado- sólo lo son en virtud del sentido, y por tanto, de cierta estructura que es mostrada por la lógica (Mounce 2007). Esto se puede entender con mucho más nitidez si observamos algunos desarrollos de la filosofía matemática, o más específicamente, de la filosofía de la lógica, como es el caso de la obra de Frege.

Frege nos propone ver que las proposiciones del lenguaje pueden concebirse como funciones matemáticas (22). Así como una función matemática, digamos $x+2$, puede tomar infinitos valores, una proposición expresada como función puede asumirse que también tiene algo equivalente a una especie de 'valor', aunque en este caso las alternativas son sólo dos: el que la proposición sea verdadera, o el que sea falsa. Por ejemplo, "x descubrió América", es una función propositiva, que es verdadera si x se refiere a Colón, y falsa si se refiere a Garibaldi. Me parece que podemos agregar a esto, que si uno tuviese conocimiento metafísico, podría a lo mejor saber decir también si el reemplazo de " $x$ " por "ángeles", en la proposición funcional "los X son inmensos", la hace a ésta verdadera o falsa. De modo que no es necesario que restrinjamos la aplicación a los meros hechos empíricos. Pero lo que en realidad nos interesa, de lo que dice en particular este carácter funcional de la proposiciones, es que mientras que la verdad o falsedad de una proposición depende de los hechos, ya sean empíricos, o metafísicos, el 'sentido', en cuanto tal, es por completo independiente, y anterior a la sentencia veritativa sobre los casos, es decir si son o no son hechos, efectivamente. La proposición planteada "Colón descubrió América" tiene sentido, independiente de que sea verdadera, o falsa, mientras que el que sea verdadera o falsa, es algo de lo cual nos enteramos posteriormente, una vez que entendemos, en efecto, el sentido de lo que en la oración se nos plantea como un hecho posible. Esto significa que la lógica, en sí misma, no nos dice nada respecto de los hechos -como diría Wittgenstein-, pero lo que sí efectúa, es que nos muestra la forma en que se articula un sentido posible acerca de los hechos enunciados en una proposición, de tal manera que luego podamos decir, por ejemplo, si son verdaderos o falsos, o en general, pensar acerca de ellos de modo predicativo.

De esta manera, a lo que arribamos es que participar de la dimensión semántica es equivalente a participar de un mundo de sentidos que reflejan una consistencia lógica. Cuando Taylor señala que el hombre es un ser de sentidos estaría diciendo, básicamente, que los seres humanos, en cuanto seres de significado, son quienes habitan este mundo o estructura de la lógica. Pero, por otro lado, es evidente que esto no es todo lo que quiere decir Taylor, pues la acepción del "sentido", en la perspectiva antropológica en que utiliza el término, y que describen sus estudios sobre la condición auto-intérprete del hombre, se refiere a los modelos de vida humanos, en la acepción de que estos encarnan ciertos valores, como lo que es significativo y bueno en la vida. De modo que la aclaración acerca de la estructura de la lógica como equivalente del trasfondo que representa el lenguaje, sólo determina un primer nivel del análisis, quedando todavía por abordar, o vincular con ello, el problema específico de la identidad, y respecto de los criterios de realización de una vida "humana". El problema gnoseológico, en Taylor, está supeditado así al problema moral, dentro de la indagación acerca del uso práctico de la razón, y es esto lo que debemos exigir en una interpretación del programa de lo que él denomina, en términos amplios, una 'teoría constitutiva' (que no se reduce, por tanto, sólo a la "constitución" del lenguaje). 
¿Cómo incorporar entonces esta posible noción del trasfondo como estructura lógica, dentro del espectro de la discusión acerca de la moral, tal como la plantea Taylor, atendiendo al problema de la orientación práctica del sujeto en base a "sentidos de vida"? Específicamente, ¿ podría considerarse que a partir de aquél tipo de trasfondo, resultara una efectiva determinación autónoma del significado, que podría, eventualmente, no incluir la intencionalidad de la condición existencial humana? No parece difícil -en base a la férrea oposición que plantea Taylor, a lo largo de toda su obra, contra los razonamientos nominalistas y conductistas-, ver que su respuesta sería que no, bajo ninguna circunstancia. Si aceptásemos - por poner el caso- que el sentido fuese algo así como un producto de la mera aplicación de unas reglas estructurales lógicas, entonces cabría pensar en una condición igualada del pensamiento humano acerca de esos significados, con el tipo de conciencia, o pensamiento de una máquina, y caeríamos con ello en la concepción avalada por el proyecto de la inteligencia artificial (23), que se opone radicalmente a la opinión que favorece Taylor. También se puede percibir la imposibilidad de esta hipótesis, en el mismo contexto de la filosofía matemática que recién antes veíamos. Como allí se indicó, pertinentemente, al referirnos a la posición de Wittgenstein (como contribuyente a la perspectiva del enfoque constitutivo), lo que éste afirma, con la independencia de la lógica respecto del mundo empírico, no es que la lógica produzca el sentido, sino sólo que lo muestra (24).

Volvamos al planteamiento de Frege para ver esto. La función propositiva "x descubrió América" tiene sentido tanto si reemplazamos x por Colón, tanto como si lo hacemos por Platón. Pero en el primer caso la entendemos además como verdadera, y falsa en el segundo. Lo mismo se puede decir de una proposición acerca del futuro: para "x llegará primero a Júpiter" hay casos en que será verdad, y otros que no, puesto que sería extraño plantear que todos los hombres llegarán a Júpiter al mismo tiempo, al menos la primera vez. No obstante, en términos de su sentido lógico: "Arturo llegará primero a Júpiter" es correcto del mismo modo que "Roberto llegará primero a Júpiter". Para nuestros efectos, lo que muestran -en lo inmediato- estos ejemplos, es que al margen de las posibilidades de sentido, que pueden cobrar las distintas situaciones hipotéticas, lo que define una vida real, humana, son las posibilidades de hechos. Que los hechos, o los estados de cosas, siempre tengan un sentido lógico (el evento "América descubrió Colón" no puede ser un hecho porque no tiene sentido) no significa que podamos subvertir este principio. Si lo que nos interesa en la acepción del 'sentido', es el 'sentido' como la forma de vida de un hombre, entonces esto implica la cuestión de los hechos en cuanto pueden ser verdaderos (vida lograda en cierta forma), o falsos (vida no lograda); es decir, de los "valores" que pueden tomar las proposiciones, o del rendimiento existencial de lo que las proposiciones dicen. Esto ha de ser así porque los hombres viven siempre en un mundo de hechos (empíricos o metafísicos), no en un mundo de proposiciones (25).

Esto no significa relativizar la necesidad para el entendimiento, en la deliberación práctica, de tener que pensar en los sentidos. Pero el propósito de este ejercicio es la conformación de una forma humana realizada bajo ciertos parámetros, no la de ciertos pensamientos. El hecho, no obstante, que sólo por medio de ellos podemos articular nuestros significados morales, no es algo que podamos despejar sin más. Podríamos preguntarnos, en efecto, cómo se vincula la condición del trasfondo concebida bajo el problema subjetivo de la orientación práctica, y la definición de la identidad implicada, en ello, por una parte, con el planteamiento del trasfondo concebido como el lenguaje o la dimensión semántica, por la otra. Estas son, como hemos visto, las vías alternativas del tratamiento que hace Taylor para enfrentar el problema del uso práctico de la razón, por parte de agentes humanos. Pero la obra de nuestro autor no avanza mucho más a este respecto, desarrollando estas perspectivas de manera integrada, con el objeto de sistematizar él mismo una teoría ontológica. El enfoque antropológico que describe su análisis se limita fundamentalmente, a este respecto, a sostener meramente un criterio para medir la plausibilidad de las distintas explicaciones posibles, sobre la base de una especie de fenomenología de la moral. En este sentido, el lugar en el que deberíamos ubicar su trabajo es al final de la línea de la tradición hermenéutica y fenomenológica contemporánea, y como indicador de algunos potenciales desarrollos a partir de la obra de los más célebres pensadores de esta corriente (26). 


\section{Notas}

(1) Nominalista cuando se trata de la crítica de las teorías sobre el lenguaje que son propias de esta tradición.

(2) El conductismo, en efecto, se centra en las conductas observables, y trata los episodios internos, o introspectivos como aspectos fantasmales, que al no ser susceptibles de investigación podemos ignorar. En este sentido -decimosse asemeja o continúa la postura empirista extrema. Incluso algunos nuevos avances conductistas, aunque admiten la posibilidad de incorporar el estudio de los estados mentales internos, lo hacen siempre considerándolos como efectos causados por el medio (Churchland). En cuanto al funcionalismo, continua evidentemente la actitud representacionalista que hemos descrito, al atender en lo específico, al carácter funcional o procedimental de los "procesos" mentales.

(3) Ya sea como ser-ahí, en la formulación antropológica del primer Heidegger, o como realización por medio del lenguaje, en la segunda etapa.

(4) Steiner subraya asimismo el aspecto material de esta circunstancia existencial (o "existenciaria" del ser en el mundo), lo que deja aún más claro la situación de la encarnación. Dice Steiner: “Dasein es 'ser ahí', y 'ahí' es el mundo: el mundo concreto, literal, real, cotidiano; ser humano significa estar sumergido, plantado, arraigado en la tierra, en la materia cotidiana, en la 'materialidad cotidiana' del mundo ('humano' viene de humus, 'tierra' en latín). Una filosofía que abstrae que pretende elevarse por encima de la 'cotidianidad' de lo cotidiano, es una filosofía vacía: no puede decirnos nada del sentido de ser, de dónde está y qué es el Dasein". Dicho de otro modo, el mismo hecho de ser un ser humano, implica ser "mundanamente", o ser "en el mundo" (Steiner 1999:160).

(5) Esto es, que lo registrado por nuestra conciencia, como evento de conocimiento, es un mero hecho, desprovisto de su carga evaluativa, o de valor.

(6) Más adelante Merleau Ponty sintetiza esta idea diciendo que este reduccionismo significa que frente a ciertas propiedades fenomenológicas de nuestra experiencia del cuerpo -como el "inacabamiento del cuerpo", o su "presentación marginal" - lo que instala la postura objetivista (mecánica o desvinculada) es que no puedan ya considerarse como "rasgos de estructura del cuerpo mismo", y pasen a convertirse, en efecto, en los "caracteres distintivos de los contenidos de conciencia que componen nuestra representación del cuerpo".

(7) Impresiones "de la sensación" como opuestas a las “de reflexión".

(8) En el espacio psíquico, en la res cogitans.

(9) "So what is thinking? It is assembling ideas, properly the assembling of clear and distinct ideas, and according to the way components of the world are assembled".

(10) Churchland sintetiza el argumento de Wittgenstein en los siguientes términos: "Supongamos que se intenta otorgarle un significado a un término ' $W$ ' solamente asociándolo con una sensación que se experimenta en determinado momento. En un momento posterior, al experimentar una sensación, uno puede decir 'Se trata de otro $W^{\prime}$, pero ¿cómo se puede determinar que uno ha usado el término correctamente en esta ocasión? Tal vez uno no recuerde bien la primera sensación, o despreocupadamente ve una estrecha semejanza entre la segunda y la primera aunque en realidad sólo existe un parecido débil y distante. Si el término ' $W$ ' no tiene ningún tipo de conexiones de sentido con otros fenómenos, como por ejemplo con ciertas causas y/o efectos corrientes del tipo de sensación de que se trata, entonces, no habrá absolutamente ningún modo de distinguir entre el uso correcto de 'W' y su uso incorrecto. Pero un término cuya aplicación correcta escapa permanentemente a la posibilidad de determinación es un término que no tiene sentido. Por lo tanto, un lenguaje necesariamente privado es algo imposible" (Churchland 1999:90).

(11) Es importante esta aclaración para indicar que esta reflexión no se identifica con el mero procedimiento racional, que podría asimilarse con las pretensiones del racionalismo, sino que se refiere, en efecto, a una especie 
de "actitud reflexiva", que designa la capacidad de introspección serena, abstraída del "océano de sensaciones" y que incluye otras formas de pensamiento, además de estrictamente racional, como por ejemplo, el pensamiento artístico.

(12) "The language I speak, the web which I can never fully dominate and oversee, can never be just my language, it is always largely our language".

(13) "Language is fashioned and grows not principally in monologue, but in dialogue, or better, in the life of the speech community".

(14) "By Import I mean a way in which something can be relevant or of importance to the desires or purposes or aspirations or feeling of a subject".

(15) "We would then have, it seems, a complete structural analogue of fear, including the import-recognition of danger and the flight".

(16) "But things can have this kind of meaning only for a subject in whose form of life there figures an aspiration to dignity, to be a presence among men which command respect".

(17) Una prueba de la subjetividad del hecho es que podríamos pensar perfectamente en una cultura distinta para cuya óptica estas propiedades no sean vergonzosas en un hombre. Esto no significa que la dimensión moral del ser humano sea toda subjetiva, lo que contradiría la opinión de Taylor. Hay por cierto objetividad en la moral, como quiere indicar nuestro autor con el concepto de import, es decir, propiedades objetivas, o propiedades de los objetos morales reales, concretos. Pero lo objetivo no es la prescripción, sino la condición moral del hombre.

(18) "For the shameful is not a property which can hold of something quite independent of the experience subjects have of it. Rather, the very account of what shame means involves reference to things -like our sense of dignity, of worth, of how we are seen by others- which are essentially bound up with the life of a subject of experience".

(19) No podemos adentrarnos aquí en una explicación pormenorizada del concepto de las distinciones cualitativas, en la obra de Taylor. No obstante, podríamos definirlas concisamente como aquellos juicios discriminativos que efectuamos los seres humanos entre distintas opciones de valores alternativos. Es decir, cuando, en el marco de nuestra facultad de agencia, buscamos obtener una determinación específica en el pensamiento de aquellas cosas -vivencias, o situaciones- que sometemos al parámetro de las valoraciones éticas, y bajo las cuales concebimos respectivamente un objeto particular de ellas en nuestro entendimiento. El trasfondo de esta clase de discernimiento "cualitativo" es lo que Taylor denomina los "marcos referenciales".

(20) "Thus man is a language animal (...) because what we consider the essential human concerns are disclosed only in language, and can only be the concerns of a language animal".

(21) Tomo este término de la "machina sapiens" del libro de Campbell La máquina increíble.

(22) Esta consideración es posible, en el fondo, porque como explica, una función a diferencia de un mero número es una expresión incompleta, o "no saturada", lo mismo que la estructura lógica de una proposición en la que podemos colocar distintos objetos. Frege llama a x, el argumento de la función. Luego dice: "Me interesa señalar que el argumento no forma parte de la función, sino que constituye, junto con la función, un todo completo; pues la función, por sí sola, debe denominarse incompleta, necesitada de complemento, o no-saturada. Y ésta es la diferencia de principio que hay entre las funciones y los números. Y por esta naturaleza de la función se explica que, por una parte, reconozcamos la misma función en " $2 \times 1^{3}+1^{\prime \prime} y$ " $2 \times 2^{3}+2$ ", a pesar de que estas expresiones se refieran

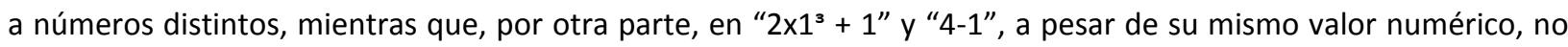
encontramos la misma función" (Frege 1985:23). 
(23) Podríamos por ejemplo, pensar en una base filosófica, en este sentido, basada en la obra de Chomsky. En el marco de su gramática generativa, Chomsky constata que a pesar de que las posibilidades de oraciones, que constituyen una determinada lengua, es probablemente infinita, cada una de ellas es una secuencia finita, compuesta de un número finito de fonemas. Luego si seguimos la operación que hace Chomsky, de tomar la gramática de una lengua como si fuera una teoría de su estructura, y definimos una lengua como "el conjunto de oraciones generada por la gramática" (Peregrín Otero 1974:XXXIV), entonces podríamos llegar a pensar de hecho en la posibilidad de ir discriminando entre oraciones finitas con sentido, y otras sin sentido, las que podríamos ir descartando, hasta llegar, en algún momento, a formular una especie de programa que en base a este criterio lógico, nos de la pauta -o el 'algoritmo'- del sentido.

(24) Esto queda bien claro al considerar la forma en que Wittgenstein propone concebir a la proposición como una figura. Según Hintikka un supuesto fundamental de esta teoría figurativa es que las oraciones verdaderas son algo así como representaciones isomórficas de la realidad de la que hablan. No es que la figura -análoga de la proposición- que aparece, por ejemplo, en una pintura, "le diga" al hombre de lo que trata, sino que esto es algo que puede ver el hombre "con" la figura (Mounce). La figura, como la proposición lógica, le muestra ese sentido.

(25) Podría plantearse esto mismo, tal vez, atendiendo a la "temporalidad" o "finitud" de la vida humana, frente a la infinitud de los sentidos potenciales. Así, por ejemplo, un hombre, en el total de su vida, no podría terminar de contar todos los números posibles, los que denotan ciertamente, cada uno, un sentido de algo. Aquí radica un factor de atracción de la concepción del ser-ahí que propone Heidegger como un ser de posibilidades.

(26) En general, la mayoría de los estudios relevan la adscripción de Taylor ya sea al pensamiento de Hegel, o de Aristóteles. Aunque esto es correcto, lo que aquí se ha visto, en particular, es que la reposición del proyecto filosófico de una ética de la vida buena, en la actualidad, según Taylor, depende también en gran medida de un mayor desarrollo y profundización (tal vez con contenido especulativo) de las perspectivas de la fenomenología y la hermenéutica. El rescate del enfoque práctico de la filosofía aristotélica, por parte de Heidegger (es decir la puesta en escena de ese enfoque, pero ahora en un marco fenomenológico), tal como lo han mostrado las investigaciones de Alejandro Vigo, y de Franco Volpi, pueden ser indicios en esta misma dirección.

\section{Bibliografía}

Benedicto Rodríguez, R. 2004. Charles Taylor: identidad, comunidad y libertad (Tesis Doctoral). Valencia: Universitat de Valencia.

Berlin, I. 2000. Vico y Herder. Madrid: Cátedra.

Churchland, P. 1999. Materia y conciencia. Barcelona: Gedisa.

Frege, G. 1985. Estudios sobre semántica. Buenos Aires: Orbis-Hyspamerica.

Heidegger, M. 1968. El ser y el tiempo. México: Fondo de Cultura Económica.

Merleau-Ponty, M. 1957. Fenomenología de la percepción. México: Fondo de Cultura Económica.

Mounce, H.O. 2007. Introducción al Tractatus de Wittgenstein. Madrid: Tecnos.

Peregrín Otero, C. 1974. Introducción a Chomsky. En: Chomsky, N. Estructuras sintácticas. México: Siglo XXI.

Steiner, G. 1999. Heidegger. México: Fondo de Cultura Económica.

Taylor, C. 1985. Philosophical Papers: Volume 1, Human Agency and Language. Cambridge: Cambridge University Press. 
Medina, C. 2014. La fenomenología del lenguaje y el concepto de la razón práctica en el pensamiento de Charles Taylor Cinta moebio 50: 53-69

Taylor, C. 1995. Philosophical arguments. Cambridge, Mass.: Harvard University Press.

Taylor, C. 1997. Argumentos filosóficos. Barcelona: Paidós.

Recibido el 28 ene 2014

Aceptado el 14 jun 2014 